\title{
Treatment of peritoneal dissemination of gastric cancer with sequential methotrexate and 5-fluorouracil
}

\author{
Toshiro Konishi ${ }^{1}$, Tamaki Noie ${ }^{1}$, Junji Yoshida ${ }^{2}$, Kenichi Mafune ${ }^{3}$, and Masatoshi Makuuchi ${ }^{3}$ \\ ${ }^{1}$ Department of Surgery, Kanto Teishin Hospital, 5-9-22 Higashi-Gotanda, Shinagawa-ku, Tokyo 141-0022, Japan \\ ${ }^{2}$ Department of Thoracic Oncology, National Cancer Center Hospital East, Chiba, Japan \\ ${ }^{3}$ Second Department of Surgery, Faculty of Medicine, the University of Tokyo, Tokyo, Japan
}

\begin{abstract}
:
Background. Pretreatment with methotrexate (MTX) followed by 5-fluorouracil (5-FU) (sequential MTX and 5-FU), exerts biochemical modulation in which the antitumor effect of 5-FU is enhanced by the preceding MTX. Since the early 1980s, the sequential MTX/5-FU regimen has been employed clinically for gastric cancer in Japan, with high response rates for undifferentiated type gastric carcinoma.

Methods. Peritoneal dissemination is more frequent in undifferentiated type carcinoma, we therefore employed sequential MTX and 5-FU for the treatment of 39 gastric cancer patients with peritoneal dissemination. We also investigated MTX levels in blood and ascitic fluid during the treatment.

Results. Partial response (PR) was achieved in 6 of 26 patients $(23 \%)$ who had evaluable lesions. PR lesions included abdominal wall tumors, primary gastric foci, inguinal lymph node, and rectal stenosis. Ascites was eliminated in $\mathbf{5 0 \%}$ of the patients, and pleural effusion disappeared in 2 patients.

Conclusions. In our study, sequential MTX and 5-FU had low toxicity and was beneficial for advanced gastric cancer patients with peritoneal dissemination. Further investigation of this treatment as induction and postoperative adjuvant chemotherapy for Borrmann type 4 gastric cancer seems warranted.
\end{abstract}

Key words: peritoneal dissemination of gastric cancer, biochemical modulation, sequential methotrexate and 5fluorouracil

Offprint requests to: T. Konishi Received for publication on Feb. 8, 1999; published on March 15,1999

\section{Introduction}

Methotrexate (MTX), a folic acid antagonist, inhibits dihydrofolate reductase (DHFR), suppresses folates (FH2) reduction, and decreases reduced form folates (FH4) [1]. Through this mechanism, MTX exerts an antitumor effect by inhibiting DNA synthesis. However, monotherapy with MTX for gastric cancer is not sufficiently effective [2], and it has been rarely reported in the literature. At present, mainstream chemotherapy for gastric cancer employs combinations of multiple agents. MTX is used, usually in combination with other drugs such as 5-fluorouracil (5-FU). MTX pretreatment followed by 5-FU (sequential MTX and 5-FU), proposed by Bertino et al. in 1977 [3], exerts biochemical modulation, in which the antitumor effect of 5-FU is enhanced by the preceding MTX.

Since the early 1980s, the sequential MTX/5-FU regimen has been employed for gastric cancer patients in Japan, and showed good results [4]. The efficacy of sequential MTX and 5-FU in gastric cancer varied according to the histologic type. Our results in 64 inoperable gastric cancer patients showed that all 9 responders had adenocarcinoma of the poorly differentiated type, and the response rate in patients with this type of carcinoma was $32 \%$ [4]. Since that report, the high efficacy of this chemotherapy regimen in undifferentiated gastric carcinoma has become widely recognized and is now generally accepted.

Although the efficacy of surgical intervention in gastric cancer patients has improved markedly in recent years, the outcome in patients with metastasis and postoperative recurrence resulting from peritoneal dissemination remains difficult to improve. Peritoneal dissemination is more frequent in patients with undifferentiated carcinoma than in those with differentiated carcinoma [5]. Since sequential MTX and 5-FU is effective for undifferentiated carcinoma, we employed this therapy for the treatment of gastric cancer patients with 
peritoneal dissemination. We describe the promising results of this chemotherapy in patients with cancerous pleuroperitonitis. We also report MTX levels in blood and ascitic fluid in these patients.

\section{Patients and methods}

\section{Patients}

We treated 39 gastric cancer patients with peritoneal dissemination. Twenty-one had recurrent disease after gastrectomy and 18 had primary inoperable advanced gastric cancer. Twenty-six of the 39 patients had measurable lesions (Table 1); 16 of the 39 had malignant ascites, and 2 of the 39 had malignant pleural effusion.

\section{Sequential MTX and 5-FU}

We used an intermediate-dose program, which consisted of MTX $\left(100 \mathrm{mg} / \mathrm{m}^{2}\right)$ given by intravenous bolus injection, followed $1 \mathrm{~h}$ later by $5-\mathrm{FU}\left(800 \mathrm{mg} / \mathrm{m}^{2}\right)$ given as 1-hour intravenous drip infusion. All patients in the intermediate-dose program $(n=24)$ received eight consecutive intravenous injections of $15 \mathrm{mg}$ calcium leucovorin at the 6-h intervals from $24 \mathrm{~h}$ after the MTX administration. To reduce the adverse effects of MTX, $250 \mathrm{mg}$ of acetazolamide was given orally for urine alkalization prior to each treatment. Intravenous infusion of sodium bicarbonate during 5-FU administration was also added for the same purpose. Low-dose treatment, which consisted of $30 \mathrm{mg} / \mathrm{m}^{2}$ of MTX followed by $600 \mathrm{mg} / \mathrm{m}^{2}$ of $5-\mathrm{FU}$ after an interval of $3 \mathrm{~h}$, was given to poor-risk patients or to patients treated in the outpatient clinic $(n=15)$ along with the same urine alkalization as in the intermediate-dose treatment. Low-dose treatment was also followed by two oral or intravenous doses of $15 \mathrm{mg}$ leucovorin on the following day.

\section{Response}

It is often difficult to determine therapeutic efficacy for peritoneal dissemination with imaging examinations.

Table 1. Main target lesions

\begin{tabular}{lc}
\hline & No. of patients \\
\hline Primary gastric focus & 16 \\
Rectal stenosis & 3 \\
Abdominal wall tumor & 3 \\
Intraabdominal tumor & 2 \\
Lymph node & 1 \\
Liver & 1 \\
No of patients with measurable lesions & 13 \\
Total & 39 \\
\hline
\end{tabular}

We therefore evaluated the efficacy of the sequential MTX and 5-FU based on the changes not only in the disseminated lesions but also in the primary sites and foci other than the dissemination.

On the image evaluation, standard criteria were used to define the response. Complete response (CR) was defined as $100 \%$ disappearance of all measurable tumors and the absence of tumor-related symptoms for a minimum of 4 weeks. Partial response (PR) was recorded as $\geq 50 \%$ reduction in the product of the two longest perpendicular diameters of measurable tumors, persisting for at least 4 weeks in the absence of new lesions. No change (NC) was defined as $<50 \%$ decrease or $<25 \%$ increase in the product of the two longest perpendicular diameters of all measurable lesions. In the $\mathrm{NC}$ category, $<50 \%$ reduction in the tumor was designated as minor response (MR). Progressive disease (PD) was defined as $\geq 25 \%$ increase in the product of the two perpendicular diameters of a measurable tumor.

\section{Measurement of MTX in blood and ascitic fluid}

Serum MTX level was determined in 20 patients with advanced gastric cancer 2, 6, 24 and $48 \mathrm{~h}$ after MTX administration in the intermediate-dose treatment. In 2 patients treated with the low-dose program, the serum MTX level was determined 2, 6, 24, and 48 h after MTX administration. Sixteen of the 20 patients in the intermediate-dose group had no ascites, but the remaining 4 had ascites owing to cancerous peritonitis. In 2 of the 4 patients who had ascites, the MTX levels in ascitic fluid as well as in blood were determined during the first and second treatment courses.

\section{Results}

\section{Therapeutic efficacy for peritoneal dissemination}

Of the 39 patients with metastasis caused by peritoneal dissemination treated by the sequential MTX and 5-FU, 26 patients had measurable lesions and PR was achieved in $6(23 \%)$. The PR lesions included abdominal wall tumor (3 patients), primary gastric focus (2 patients), inguinal lymph node (1 patient), and rectal stenosis (1 patient). Ascites disappeared in 8 of the 16 patients $(50 \%)$. In both of the patients who had pleural effusion, this was also eliminated. In the patients in whom ascites disappeared, the effect was evident after the first or second therapy. The efficacy of sequential MTX and 5-FU on fluid retention lasted 3-8 months in patients with ascites, but only about 2 weeks in both of the patients with pleural effusion.

Concerning toxicity, leukopenia, with a white blood cell count of less than $2000 / \mathrm{mm}^{2}$ was observed in 4 of the 
39 patients. Grade 4 diarrhea and grade 3 nausea and vomiting was seen in 1 patient each, but the treatment was well tolerated in all patients with no toxicity.

\section{MTX levels in serum and ascites}

We evaluated serum MTX levels in 16 patients without ascites who received 38 courses of the intermediatedose program in total. The mean serum MTX levels 2, 6, 24 , and $48 \mathrm{~h}$ after bolus i.v. administration of $100 \mathrm{mg} / \mathrm{m}^{2}$ MTX were $5 \times 10^{-6} \mathrm{~mol} / 1,1 \times 10^{-6} \mathrm{~mol} / 1,5 \times 10^{-8} \mathrm{~mol} /$ 1 , and $2 \times 10^{-8} \mathrm{~mol} / \mathrm{l}$, respectively (Fig. 1). In contrast, the mean serum MTX levels 2, 6, 24, and 48 h after MTX administration of eight intermediate-dose courses in 4 patients with pleural effusion or ascites were $7 \times$ $10^{-6} \mathrm{~mol} / 1,2 \times 10^{-6} \mathrm{~mol} / 1,9 \times 10^{-8} \mathrm{~mol} / 1$, and $5 \times$ $10^{-8} \mathrm{~mol} / \mathrm{l}$, respectively. In the low-dose group, the mean serum MTX levels 2, 6, 24, and $48 \mathrm{~h}$ after i.v. administration of MTX were $2 \times 10^{-6} \mathrm{~mol} / 1,1.5 \times 10^{-7} \mathrm{~mol} / 1,2.7 \times$ $10^{-8} \mathrm{~mol} / \mathrm{l}$, and $2 \times 10^{-8} \mathrm{~mol} / \mathrm{l}$, respectively.

In the two patients with ascites in whom the MTX levels in ascitic fluid were determined, the ascites levels exceeded those in serum at $6 \mathrm{~h}$ after the first dose of MTX and stayed higher until $72 \mathrm{~h}$ (Figs. 2, 3). However, after the first dose of sequential MTX and 5-FU, the ascites decreased, and after the second course, the MTX levels in blood and ascites remained lower than in the first treatment.

\section{Discussion}

In a Japanese phase II clinical study of sequential MTX and 5-FU in gastric cancer patients, the low- and

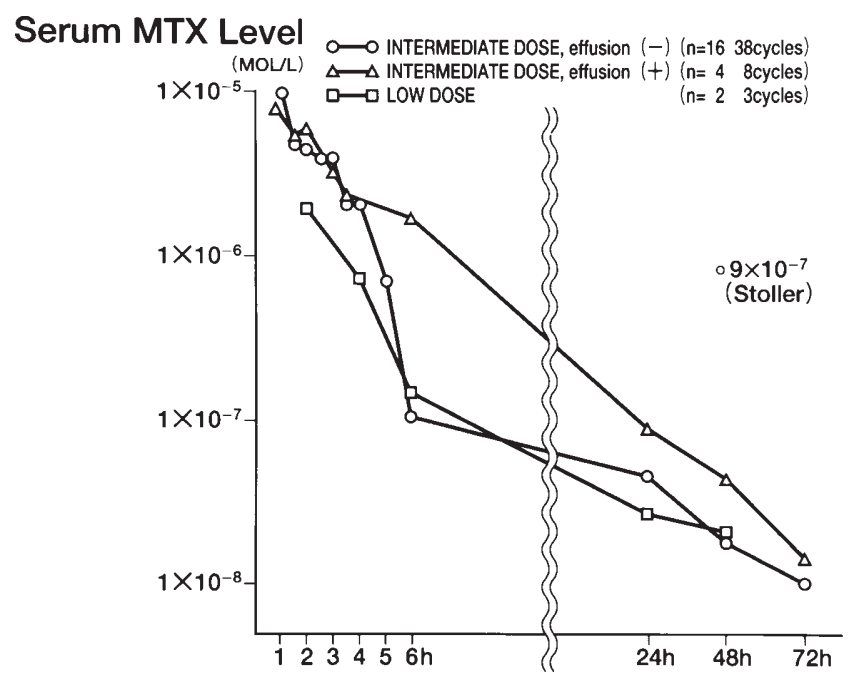

Fig. 1. Serum methotrexate $(M T X)$ levels after treatment. (Circles, Intermediate-dose in 16 patients with out pleural effusion (38 cycles); triangles, intermediate-dose in 4 patients with ascites or pleural effusion (8 cycles); squares, low-dose in 2 patients ( 3 cycles) intermediate-dose regimens achieved high response rates, of $23 \%(13 / 56)$ and $41 \%(15 / 37)$, respectively [6]. In another study of the intermediate-dose regimen in patients with advanced or recurrent gastric cancer, the overall response rate was $18 \%(10 / 56)$, while it was $19 \%$ $(5 / 26)$ for differentiated carcinoma and $17 \%(5 / 30)$ for undifferentiated carcinoma, showing no significant difference according to histologic type [7]. However, in our previous study, this therapy was highly effective for undifferentiated carcinoma, with a response rate of $32 \%$ $(9 / 28)$, while there was no response in differentiated carcinoma (0/10) [4], and the high efficacy of this regimen in undifferentiated gastric carcinoma is now generally accepted. The antitumor effect of 5-FU is reported to be enhanced by modulation of both RNA function perturbation [8] and DNA synthesis inhibition [9]. The

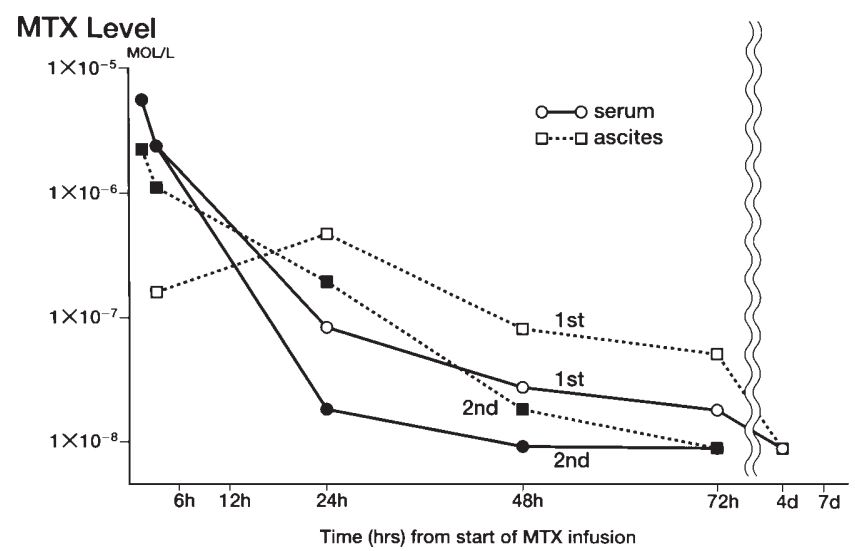

Fig. 2. MTX levels in serum and ascites: case 3. MTX levels in serum (circles) and in ascites (open squares) in the first course decreased in both serum (dots) and ascites (solid squares) in the second course, given 1 week after the initial course, with clinical signs of diminishing ascites

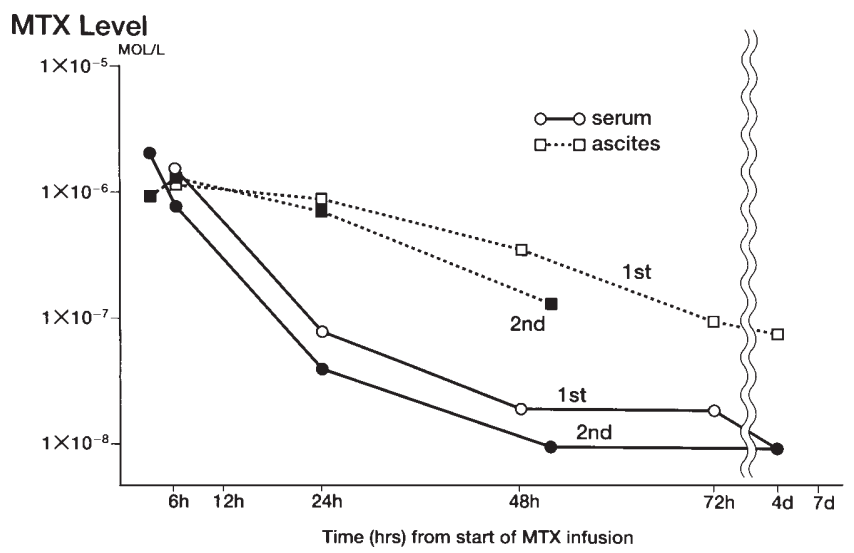

Fig. 3. MTX levels in serum and ascites: case 4. MTX levels in serum (circles) and in ascites (open squares) in the first course decreased in both serum (dots) and ascites (solid squares) in the second course, given 1 week after the initial course, with clinical signs of diminishing ascites 
higher efficacy of this sequential therapy in undifferentiated carcinoma may be explained by the difference in activity of two enzymes, thymidylate synthetase and thymidine kinase, in the different histologic types of gastric cancer [10].

Since the sequential MTX and 5-FU was highly effective for undifferentiated gastric carcinoma, we employed the therapy in 39 patients who had advanced or recurrent gastric carcinoma with metastasis resulting from peritoneal dissemination, which is common in patients with undifferentiated gastric carcinoma. We evaluated the response both in the primary focus and in foci other than peritoneal dissemination, in the hope of achieving a more comprehensive evaluation of the therapeutic efficacy. PR was achieved in 6 of the 26 patients $(23 \%)$ who had measurable lesions. PR lesions included abdominal wall tumor, primary gastric focus, inguinal lymph node, and rectal stenosis. Ascites was eliminated in 8 of 16 patients (50\%), and pleural effusion disappeared in both of the two patients. Our study showed that sequential MTX and 5-FU had a good response rate in advanced gastric cancer patients with peritoneal dissemination.

Mizumoto et al. [11] reported a good result with sequential MTX and 5-FU in a patients with peritoneal recurrence with bilateral hydronephrosis and ascites after surgery for Borrmann type- 4 gastric cancer. Kosaka et al. [12] also employed sequential MTX and 5-FU therapy for 27 gastric cancer patients with peritoneal dissemination, and achieved a response rate of 33\% (9/ 27). When alleviation of symptoms such as ileus and ascites was included in the results, they found that this therapy was effective in $40 \%$ of the patients. They also reported that better therapeutic efficacy was achieved by an arterial infusion method (in which MTX and 5-FU were infused via an intraaortic catheter retained at the Th 9-10 level) than by intravenous infusion. Kitamura et al. [13] administered low-dose sequential MTX and 5-FU therapy by arterial infusion via an indwelling intraaortic catheter in 46 patients with advanced or recurrent gastric cancer, and reported that the treatment was effective for gastrointestinal tract stenosis. These results may encourage further study of arterial infusion of the sequential therapy in patients with small or large bowel stenosis resulting from peritoneal dissemination.

Since intravenously administered MTX passes into body fluid in patients with retention of ascitic fluid or pleural effusion, as shown in our patients, a favorable antitumor effect on peritoneal/pleural dissemination can be expected. In our series, ascites was eliminated in $50 \%$ of the patients, and there was also a disappearance of pleural effusion. However, in the patients with peritoneal dissemination but without ascites, direct intraperitoneal administration may be more effective. Takamatsu et al. [14] employed sequential MTX and 5-
FU for peritoneal metastasis of advanced gastric cancer, using intravenous infusion of 5-FU and intraperitoneal infusion of MTX, and reported its low toxicity.

Sequential MTX and 5-FU therapy generally has milder adverse effects than other types of multi-agent chemotherapy. Although the low-dose regimen infrequently causes grade- 4 marrow suppression in patients with poor performance status [13], severe adverse reactions of grade 3 or higher are rare, and the treatment can be repeated for a prolonged period in outpatient clinics. However, to reduce the adverse effects of MTX, it is desirable to concomitantly use acetazolamide and/ or sodium bicarbonate to aid in its prompt excretion from the kidney by alkalization of the urine. Combined use of MTX with nonsteroidal anti-inflammatory drugs should be avoided [15]. In patients with body fluid retention, MTX passes into the retained fluid, resulting in a persistently high serum level. Therefore, it is important to monitor blood MTX level to determine that it is less than $9 \times 10^{-7} \mathrm{~mol} / \mathrm{l}$ at $48 \mathrm{~h}$ after administration [16]. Based on our measurements of MTX serum levels, we believe that the administration of MTX at the intermediate or lower dose level is safe. In patients with massive ascites, however, we may use a lower initial MTX dose of $100 \mathrm{mg} / \mathrm{body}$ to reduce the related risks.

We have employed sequential MTX/5-FU as preoperative induction or postoperative chemotherapy in patients with advanced gastric cancers of poorly differentiated type, particularly diffuse infiltrating (Borrmann type 4) gastric cancers. Patients with curatively resected Borrmann type 4 treated with postoperative sequential MTX/5-FU showed a significantly better survival rate (5-year; 53\%) than our historical group treated with conventional adjuvant chemotherapy (5-year; 32\%) $(P<0.05)$ [17]. For patients with palliative resection of Borrmann type 4 gastric cancer, long-term low-dose sequential MTX/5-FU resulted in a better survival rate than the intermediate-dose program or conventional chemotherapy after the surgery [18]. Further, several patients with unresectable Borrmann type 4 cancer became resectable with this treatment.

From the viewpoint of the heterogeneous nature of cancer foci, the clinical effect of this therapy can be enhanced by combining it with other chemotherapy which is effective for cancers of higher grade differentiation. Miyashita et al. [19] reported a patients with postoperative hepatic and peritoneal recurrence. They combined sequential MTX / 5-FU and chemotherapy with arterial mitomycin $\mathrm{C}$ infusion, and achieved a survival of 27 months before the patient died, the death being attributable to another disease; autopsy revealed that the peritoneal dissemination had disappeared. A combination of sequential MTX and 5-FU treatment and intraperitoneal administration of cisplatin has also been attempted, with a favorable result [20]. 
In conclusion, sequential MTX and 5-FU is worth further investigation as an effective chemotherapy for peritoneal dissemination of gastric cancer because of its advantages: high efficacy in poorly differentiated adenocarcinoma frequently associated with peritoneal dissemination, persistent high MTX levels in ascites, and mild toxicity. As induction and postoperative adjuvant chemotherapy against Borrmann type 4 gastric cancer, sequential MTX and 5-FU should be evaluated in a prospective randomized study.

\section{References}

1. Chabner BA. Methotrexate. In: Pharmacologic principles of cancer treatment: WB Saunders; 1982:229.

2. Bruckner HW, Lokich JJ, Stablein DM. Studies of Baker's antifol, methotrexate, and razoxane in advanced gastric cancer: A gastrointestinal tumor study group report. Cancer Treat Rep 1982;66:1713-7.

3. Bertino JR, Sawicki W, Lindquist CA, Gupta VS. Scheduledependent antitumor effects of methotrexate and 5-fluorouracil. Cancer Res 1977;37:327-8.

4. Konishi T, Hiraishi M, Mafune K, Miyama T, Hirata T, Mori K, et al. Therapeutic efficacy and toxicity of sequential methotrexate and 5-fluorouracil in gastric cancer. Anticancer Res 1994;14:127780.

5. Sowa M, Kato Y, Nishimura M, Yoshino H, Kubo T, Umeyama $\mathrm{K}$, et al. Clinicohistochemical studies on type 4 carcinoma of the stomach with special reference to mucopolysaccharides and sialic acid in tumor tissue. Jpn J Surg 1989;19:153-62.

6. Murakami M, Ota K, Miyazaki T, Niitsu Y, Wakui A, Yokoyama $\mathrm{M}$, et al. Sequential methotrexate - 5-fluorouracil (MTX-5-FU) treatment for patients with advanced gastric and colorectal cancer (in Japanese with English abstract). Jpn J Cancer Chemother 1987:14:2482-90.

7. Sasaki T, Ota K, Ibayashi J, Sakata Y, Matsuoka T, Ishikawa M, et al. Randomized multicenter trial of sequential methotrexate and 5-fluorouracil versus 5-fluorouracil alone in advanced gastric cancer (in Japanese with English abstract). Jpn J Cancer Chemother 1989;16:2545-55.

8. Cadman E, Heimer R, Devis L. Enhanced 5-fluorouracil nucleotide formation after methotrexate administration; explanation for drug synergism. Science 1979;205:1135-7.
9. Fernandes D, Bertino JR. 5-Fluorouracil-methotrexate synergy: Enhancement of 5-fluorodeoxyuridylate binding to thymidylate synthase by dihydropteroylpolygluamates. Proc Natl Acad Sci USA 1980;77:5663-7.

10. Konishi T, Miyama T, Sakamoto S, Hirata T, Mafune K, Hiraishi $\mathrm{M}$, et al. Activities of thymidylate synthetase and thymidine kinase in gastric cancer. Surg Oncol 1992;1:215-21.

11. Mizumoto K, Sawada T, Furumoto T, Kawamura Y, Kishi K, Katoh K. A case report: postoperative recurrence of peritoneal dissemination of gastric cancer responding to sequential methotrexate and 5-FU (5-fluorouracil) (in Japanese with English abstract). Jpn J Cancer Chemother 1995;22:281-4.

12. Kosaka T, Tsuchiya K, Sugaya J, Yoshida S, Nakano Y, Akiyama $\mathrm{T}$, et al. A study of arterial infusion chemotherapy for gastric cancer patients with peritoneal metastasis (in Japanese with English abstract). Jpn J Cancer Chemother 1996; 23:1467-71.

13. Kitamura M, Arai K, Iwasaki Y. Arterial infusion chemotherapy for advanced gastric cancer by sequential MTX / 5-FU (in Japanese with English abstract). Jpn J Cancer Chemother 1996; 23:1464-6.

14. Takamatsu S, Maruyama M, Sugano N, Katada M, Ebuchi M. Intraperitoneal infusion therapy of MTX and 5-FU for advanced gastric cancer and its peritoneal metastasis (in Japanese with English abstract). Jpn J Cancer Chemother 1995;22:1619_ 21.

15. Thyss A, Milano G, Kubar J, Namer M, Schneider M. Clinical and pharmacokinetic evidence of a life-threatening interaction between methotrexate and ketoprofen. Lancet 1986;: I:256-8.

16. Stoller RG, Hande KR, Jacobs SA, Rosenberg SA, Chabner BA. Use of plasma pharmacokinetics to predict and prevent methotrexate toxicity. N Engl J Med 1977;297:630-4.

17. Konishi T. Treatment of scirrhous carcinoma of the stomach. Asian Med J 1996;39:291-9.

18. Konishi T, Hiraishi M, Kajiura N, Kanamaru H, Kimura T, Kouzuma T, et al. Postoperative low-dose sequential methotrexate and 5-fluorouracil in palliative resected Borrmann type 4 gastric cancer. Proceedings of Am Soc Clin Oncol 1995; 14, March, p 218.

19. Miyashita K, Kitamura M, Arai K. Effective treatment for liver metastasis and peritoneal dissemination of gastric cancer using intra-arterial therapy. A case report (in Japanese with English abstract). Jpn J Cancer Chemother 1992;19:1749-52.

20. Shibata N, Tamai M, Ikeda K. Case report of long-term survivor of advanced gastric cancer associated with peritoneal seeding successfully treated with cancer chemotherapy (in Japanese with English abstract). Jpn J Cancer Chemother 1995;22:119_ 22. 